International Review of Research in Open and Distributed Learning Volume 16, Number 4

October - 2015

\title{
Design Considerations for Integrating Twitter into an Online Course
}

Linda E. Rohr, Jane Costello, and Thomas Hawkins

Memorial University of Newfoundland

\begin{abstract}
While the use of Twitter for communication and assessment activities in online courses is not new, it has not been without its challenges. This is increasingly true of high enrolment courses. The use of a Twitter Evaluation application which leverages a Learning Management System's (LMS's) application programming interface (API) provides a solution which reduces the administrative overhead associated with tracking students' Tweets and allows the grader to focus on the assessment of the Tweets' quality. Such an application and how it improved the assessment process of grading Tweet events is described. In addition to the technological considerations, the soundness of the Twitter learning design in the course also impacts the effectiveness of the Tweet events for learning and enhancing the much-needed online social presence. Learning design considerations are also discussed.
\end{abstract}

\section{Introduction}

The education system is changing (Fisher \& Baird, 2005) and becoming more keenly focused on a student-centered, technologically-, and socially-rich environment that facilitates the formation of learning communities, ultimately promoting student engagement. Although a number of learning theories exist, social constructivism is a respected approach for designing effective learning strategies (Woo \& Reeves, 2008) for online learning. Here, the social interaction and engagement between learners and their instructors is critical. Through meaningful engagement to solve problems, analyze information, and evaluate course content, student learning is fostered (Vrasidas \& McIsacc, 1999; Woo \& Reeves, 2008). For meaningful engagement to occur however, learning opportunities, evaluation pieces, and other course materials need to be intentionally designed.

Recent advances in mobile technology have provided new venues for learning and broadened the scope of the educational environment. Innovative educational designs encourage learning in any 
desired context; students and instructors are not limited to face-to-face learning in traditional lecture environments. This flexibility in the instructional environment (Hsu \& Ching, 2012) allows the student to participate in the learning process beyond the borders of the classroom and has been shown to enhance active participation (Harrison \& Thomas, 2009). Further, micro-blogging, along with other social networking applications, are efficient for enhancing social learning (Gunawardena et al., 2009). Twitter, one of the most notable micro--blogging services, emerged in J uly 2006. As of May 2015, Twitter had over 302 million active users (Statista, 2015).

Twitter is a social media phenomenon, a dynamic, freely available resource that encourages individuals to post 140 character thoughts, ideas, or updates. This micro-blogging social networking tool provides a way of communicating with others as individuals share their own original ideas, or repost (re-Tweet) thoughts from others. Striving to compose meaningful Tweets helps learners become critical thinkers. Twitter provides an opportunity for users to create useful information and share knowledge (MacManus \& Porter, 2005).

We introduced Twitter into a large enrollment online course to increase the social presence and sense of community amongst students. This interaction was designed to increase meaning and understanding for students as users, integrated personal interpretation of course content with dynamic, online technology (Woo \& Reeves, 2008). As noted by O'Reilly (2005), the integration of curriculum with technology has changed both how educators instruct and how students learn. In many learning theories the value or importance of the student learner is paramount. Learning itself is an active process where the learner's prior experience mediates the acquisition of new knowledge (Bruner, 1987). In general, the social constructivism models for learning highlight the need for learners to connect, collaborate, and share to facilitate learning (Woo \& Reeves, 2008). As reported by Wang (2014), social software technology positively contributes to student engagement and enhances this learning process

\section{Implementation Strategy}

HKR 1000, Fitness and Wellness, is an introductory online course that is widely subscribed to by students at Memorial University. Although not applicable to degree programs offered by the School of Human Kinetics and Recreation, this elective course is selected by approximately 1000 students each year. In each of the fall, winter, and spring semesters, sections of 80 students are virtually combined into one course shell within the learning management system (LMS), Brightspace, formerly Desire2Learn (D2L). Each section of 80 students has a designated teaching assistant (TA) who initiates contact in the first few days of the course. Through a common introductory email, the TA of each section shares their appropriate contact information. Students only need to be aware of their section division when sending questions to their TA; otherwise, students are part of one learning community. Each TA, however, is responsible for grading and corresponding via email to their students only.

Course content is shared with all registered students concurrently. Content for HKR 1000 focuses on key topics for living healthy, active lifestyles, including basic principles of physical fitness, 
cardiorespiratory endurance, muscular strength and endurance, flexibility, body composition, nutrition, weight, and stress management. Clearly, content is relevant for all regardless of program of study, age, gender, etc. Even without any formal health or wellness training, all students would have some experience with physical activity and nutrition. This prior familiarity with course content lends itself well to students' learning as they attempt to bring meaning to their experiences and further deepen their knowledge (Driscoll, 2005).

With such a large number of students in the course, the Twitter design and integration was crucial for success. A number of directives about Twitter were provided to students early in the term. These directives were communicated in a variety of ways, including via email, course content, and news items. First, students were reminded that their student profiles within D2L needed to be updated with their Twitter ID handle. Students were not required to create a separate Twitter account for this course however they needed to ensure that their accounts were not private. By linking their Twitter ID handle with their D2L profile, our custom designed Tweet Analyzer could capture the appropriate Tweets. Second, students were prompted to include the hashtag HKR1000 (\#HKR1000) with the Tweets for the course. These directives were presented within the course and therefore available to students as soon as they accessed the course content. Additionally, a news item was posted during the first week of the term, reinforcing the importance of updating profiles and using the correct hashtag. The introductory email from the TA, sent during the first week of the term, also reiterated the Twitter requirements for the students. In addition to this messaging, a real time scroll of any Tweets with \#HKR1000 was included on the home page for the course. Therefore, regardless of whether a student had linked their profile or started to follow \#HKR1000, they were able to read and view all Tweets when they accessed the course.

In order to ensure the Twitter events were meaningful, careful implementation was required. During the first five weeks of the 13-week course, Twitter was solely used as a communication tool for the teaching assistants. Periodic Tweets reminding students to complete a weekly reading, an online self-check or to be healthy/ active were shared. All TAs were requested to make two to three Tweets each week. During these initial weeks many students "tested" their accounts and made practice or test Tweets to ensure everything was working properly. The real-time scroll of Tweets allowed students to check their connections independently. There were no requirements for students to make substantive comments, however, a small number of students mimicked the TA's Tweets and shared healthy living tips or observations. A reminder for students to update their Twitter profile was again circulated via Twitter during the fifth week of the semester, in anticipation of the first Tweet event.

During the fourth week of the semester, students were required to complete the first lab, the "Personal Wellness Assessment Lab." Here students were asked to reflect on their own personal wellness and identify their healthy living habits on a number of topics, including exercise/ fitness, nutrition, tobacco use, alcohol and drugs, emotional health, safety, disease prevention, etc. Responses were on a three point Likert Scale with almost always (two points), sometimes (one point) and never (zero points) as potential responses. The course instructor compiled data from this personal reflection into aggregate form. Summary tables were then posted in the course 
content area of HKR 1000. These tables, along with the course readings, formed the basis of the first Twitter event scheduled during week six where students were given an open, provocative question upon which to respond. This intentional linking of course content, and a previous assignment with the Twitter event, aimed to provide a meaningful learning experience for the students as prescribed by the constructivist learning theory (Gergen, 1999).

Directions to the students for the Twitter event are summarized below:

For each question you should make a minimum of two "Tweets": one Tweet to express your own thoughts, ideas, interpretations, etc., in relation to the week's question and a minimum of one thoughtful Tweet in response to the posts of others. Please note that responding "I agree" is not acceptable. The challenge is that you only have $\mathbf{1 4 0}$ characters at a time, so think carefully about the statement you want to make! There's a lot you can do in a limited space!

Be sure to use the hashtag \#HKR1000 in all of your responses.

Your text defines health as "the overall condition of the body or mind and the presence or absences of illness or injury." It also presents the World Health Organizations (WHO) definition, which is: "a state of complete physical, mental, and social wellbeing and not merely the absence of disease."

Your text defines wellness as "optimal health and vitality, encompassing the six dimensions of wellbeing - physical, emotional, intellectual, interpersonal, spiritual, and environmental.”

It is clear that these two concepts are inescapably connected, yet wholly distinct unto themselves.

With this in mind, review the tables/charts provided that summarize some of the health and wellness related questions from the lab that you just completed. Please take a few moments to review each of the diagrams carefully and then Tweet a response to the question "Based on your readings of the provided tables/charts what can you conclude about the overall health and wellness of people in your HKR 1000 class?"

Teaching assistants graded the Tweets, with each Twitter event worth 5\%. Each original and response Tweet was graded out of 2.5 with 1.5 marks for completion and 1 mark for quality. Specific grading instructions for quality included "the student needs to make a comment that shows they thought about the topic. Example '68\% of our class gets enough sleep' would not get full marks, but '68\% of our class gets enough sleep which can help people focus and be more alert all day' would get a better mark." The better mark was awarded for answers that showed the students read the material, analyzed it and the class data, and attempted to make a link between the two, maintaining the 140 character limit.

To facilitate grading our Tweet Analyzer leveraged both the Twitter API (https://dev.twitter.com/rest/public) and the Valence API available from D2L (http://docs.valence.desire2learn.com/) to gather information both from Twitter and D2L to build a custom application that could make the grading of these Tweets a reasonable task. 
Previous uses of Twitter necessitated students and instructors following each other, instructors tracking down student Twitter identities, and constantly reviewing the hashtag feed to determine if students had Tweeted for the assessment. This was a laborious activity for the instructor, sometimes involving several hundred hours of painstaking effort. A custom application was built, leveraging the LMS technology, which reduced the non-assessment overhead activity. Our custom application pulled all Twitter IDs for each of the students from D2L, and stored them in a database along with the corresponding section of the student. Next, it regularly queried each of the Twitter handles and downloaded each Tweet that matched the specified hashtag. TAs then used the widget within D2L to browse through the sections of students and view their list of Tweets in detail. Also recorded with the Tweet were links to any attachments, such as URLs and images, as well as, other hashtags and mentions. A link was provided for TAs to view the Tweet directly on the Twitter website in context with other Tweets if the Tweet was part of a discussion.

The student view for this D2L widget included feedback regarding which Twitter ID was read from their D2L profile along with an update regarding the last time their account was checked for Tweets. If the student had not provided a Twitter ID, or if their account was unable to be read because it was configured as private, the student was notified of this as well. TAs and instructors could see, at a glance, which students had not entered their Twitter ID in their LMS user profile and contact them via internal mail. For both TAs and students, a list of Twitter events was presented along with the required hashtag, and start and end dates. A summary of Tweets relating to a Tweet event could be downloaded as a CSV file.

Scheduled during week 11 of the semester, the second Twitter event focused on a nutrition video and course content. Here again, students were required to make one original and one response Tweet guided by the questions (What did you learn about the nutritional value of food that you didn't know before?; What did you learn about the role of vitamins/ supplements in a healthy diet? and; What are your thoughts about the controversy surrounding vitamin supplementation?) posed about the video. Grading was similar to the first Twitter event.

\section{Intentional Design Considerations}

A number of intentional course design considerations were incorporated into HKR 1000 in order to make the use of Twitter effective and meaningful. Students were provided with consistent messaging about the use of Twitter within the course, and this messaging was repeated over the first four weeks of the term. Multiple sources of information about Twitter accounts, updating D2L student profiles, and the use of \#HKR1000, encouraged compliance with the Twitter requirements for the course.

The real time Twitter feed within the course shell provided a strategy for students to independently check if their posts were being recognized and ensuring their contributions to HKR1000 were visible to all. This feed also provided a concise summary of all Tweets for the course. 
During week 4 of the course, all students completed the Personal Wellness Assessment. This assessment was a short survey, asking students about their daily health and physical activity. Summary data from this assessment was provided to all students during week 5 and informed the first Twitter event during week 6. Coupling of the course content and previous assignment within the Twitter event encouraged students to link content to life and helped enrich the learning environment.

The two Twitter events were spaced 4 weeks apart in the 13-week course. Feedback on the first event was provided within 7 days of the event closing. For those few students who failed to follow the instructions regarding setting up their D2L profile or failed to include the \#HKR1000 in their Tweets, the grading from the first event provided feedback in ample time for them to correct their mistakes prior to the second Tweet event.

Both Twitter events encouraged critical thinking and personal reflection. In particular, the second event focused on a video that some might label as "controversial," or at least, a video with an atypical perspective on nutrition and healthy eating. However, the course text, the other resource for the event, presents helpful, factual and a more traditional approach to the topic. Together these two resources guided the event and provided rich content for reflection and critical thinking.

Other design considerations emerged from this Twitter event. Namely, students were encouraged to use a Twitter account specifically for academic pursuits; opportunities for participants to practice Tweeting were set up prior to the first formal Tweet event; expectations relating to Tweets and grading were clearly outlined; Tweet events were closely tied to course content in terms of topic and timing, and other course assignments and activities; LMS features were used to make the Tweets accessible and reduce assessment overhead for TAs and instructors; the Twitter hashtag and feed were available all semester for other communication needs; displaying of students' images in the Twitter feed allowed students to recognize each other and see each other as real.

A few challenges occurred that impacted the learning design. These were readily surmounted. Some, such as effort to track and assess Tweets, were discussed previously. Other issues were effectively addressed as they arose. For example, some students did not have their Twitter identity recorded in their LMS user profile prior to the scheduled Tweet events. The TA or instructor contacted these students to remind them that this information was needed in order for them to be assessed for this activity. Students mistyped their Twitter identity in their LMS user profile causing their Tweets to be unreachable from within the LMS. A few students chose not to participate in the Twitter events. They did not contact their TA or instructor and no reason was given. The Tweet Analyzer needed refining to capture some of the exceptions encountered when downloading and matching Tweets to students' LMS ID. These challenges afforded the opportunity to build a more robust analyzer. The initial attempt to write a Tweet collector used 
the Twitter search/Tweets API, which turned out to not be appropriate. The search/Tweets API only includes 6 to 9 days of Tweets, and the Tweets that are included are based upon an unpublished formula designed to promote "interesting" or "important" Tweets and filter out bots and spammers. Using this API we found that many students Tweets would be missed until they became more prolific Tweeters and Twitter then allowed their Tweets to appear in search. This realization caused us to move to the statuses/ user_timeline API instead, which returns all Tweets for a given Twitter handle.

One challenge created through the online learning environment is that the sense of community is often degraded. Students, particularly those registered in large enrollment online courses, report difficulties connecting with fellow classmates, teaching assistants, and course instructors. An online survey available to all students in HKR1000 at the end of each of two semesters assured course design specialists that a strong sense of community was present in HKR1000, and was positively impacted by the use of Twitter, both as an assessment tool and as a means of communication.

As a popular online elective it is not uncommon for a term enrollment in HKR1000 to exceed 400 students. To ensure quality and timely feedback for students, and to ensure each student feels connected to course instructors each group of 80 students was assigned to specific teaching assistants. This first point-of-contact provided students with clear connection to guidance and helped to ensure consistent evaluation throughout the term. In all semesters, Tweets were shared with all students/TA increasing the learning community base and providing more opportunities for enhanced collaboration.

\section{Conclusion}

Incorporation of Twitter events for assessment is can be an effective means to encourage engagement and community in large online classes. Care in a course's learning design is important. From a learning design perspective, Twitter's use ought to be carefully considered for suitability to the course's philosophy, content, and participants' capabilities. It should be closely tied to other class activities and content, both in terms of topics and timing. Its reason for being used ought to be communicated to students, be it for communication of course logistics, reporting on current events, or other assessment-related activities. Using applications to facilitate the

aggregation and evaluation process of the Tweet events affords instructors and TAs more time to focus on other course matters. 


\section{References}

Bruner, J . (1987). Prologue to the english edition. In R. W. Rieber \& A. S. Carton (Eds.), The Collected Works of L. S. Vygotsky, Volume 1, Problems of General Psychology. New York, NY: Plenum.

Driscoll. M. P. (2005). Psychology of learning for instruction. (3 $3^{\text {rd }}$ ed.). New York: Pearson.

Fisher, M., \& Baird, D.E. (2005). Online learning design that fosters student support, selfregulation, and retention. Campus-Wide Information Systems, 22(2), 88-107.

Gergen, K. J . (1999). An invitation to social construction. Thousand Oaks, CA: Sage Publications.

Gunawardena, C. N., Hermans, M. B., Sanchez, D., Richmond, C., Bohley, M., \& Tuttle, R. (2009). A theoretical framework for building online communities of practice with social networking tools. Educational Media International, 46(1), 3-16.

Harrison, R., \& Thomas, M. (2009). Identity in online communities: Social networking sites and language learning. International J ournal of Emerging Technologies and Society, 7(2), 109-124.

Hsu, Y., \& Ching, Y. (2012). Mobile microblogging: Using twitter and mobile devices in an online course to promote learning in authentic contexts. The International Review of Research in Open and Distance Learning, 13(4), 211-227.

MacManus, R., \& Porter, J . (2005). Web 2.0 for design: Bootstrapping the social web. Retrieved from: http:// www.digital-web.com/articles/web_2 for_designers

O'Reilly, T. (2005). What is web 2.0: Design patterns and business models for the next generation of software (Online). Retrieved from http:// oreilly.com/ web2/archive/ whatis-web-20.html

Statista. (2015). Number of monthly active twitter users. Retrieved from: http:// www.statista.com/statistics/282087/ number-of-monthly-active-twitter-users/

Vrasidas, C., \& McIsaac, M.S. (1999). Factors influencing interaction in an online course. American J ournal of Distance Education, 13(3), 22- 36.

Wang, S. (2014). Collaboration factors and quality of learning experience on interactive mobile assisted social e-learning. The Turkish Online J ournal of Educational Technology, 13(2), 24-34. 
Woo, Y., \& Reeves, T. (2008). Interaction in asynchronous web-based learning environments: Strategies supported by educational research. Journal of Asynchronous Learning Networks, 12(3-4), 179-194.

(C) Rohr, Costello, and Hawkins

\section{Athabasca University $\mathbf{I}$}

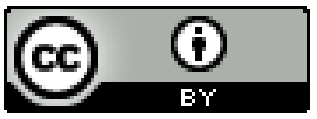

